\title{
Meningokokken-Impfung
}

\section{Früher Schutz gegen gefährliche Keime}

\begin{abstract}
Meningitis gilt als eine der prognostisch ungünstigsten Erkrankungen. Seit zwei Jahren wird in Deutschland nun die Impfung gegen Meningokokken C empfohlen. Möglicherweise steht in absehbarer Zeit auch eine Vakzine gegen den viel häufigeren Erreger Serotyp B zur Verfügung.
\end{abstract}

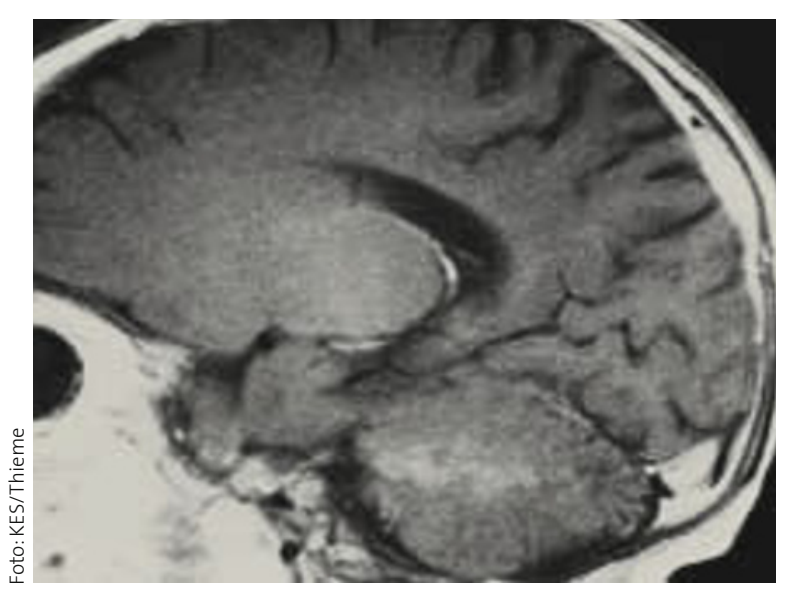

ren einen neuen spezifischen Impfstoff entwickelt. Ausgehend vom Genom eines pathogenen Meningokokkenstamms der Serogruppe B mit 570 kodierenden Sequenzen konnten gentechnologisch 350 Proteine exprimiert werden, erklärte Liese. In weiteren Prozessen wurden daraus vier immunogene Oberflächenantigene isoliert und für einen Konjugatimpfstoff entwickelt. Erste Ergebnisse einer Phase-II-Studie konnten in diesem Jahr auf dem ESPID-Kongress präsentiert werden: $100 \%$ der untersuchten Säuglinge zeigten nach drei Impfungen (inkl. Post-Booster-Impfung) einen Immunschutz gegen zwei der Vakzinantigene, 96\% bildeten eine Immunantwort gegen das dritte Antigen aus. Die Nebenwirkungsraten entsprachen den im ersten Lebensjahr routinemäßig durchgeführten Impfungen.

— Die Einführung der siebenvalenten Pneumokokkenimpfung hat in den USA zu einem deutlichen Rückgang der Menigitiden geführt, die durch Streptococcus pneumoniae verursacht waren. Eine Auswertung aus 20 USamerikanischen Notaufnahmestationen zeigte, dass nur noch 33\% der Meningitisfälle auf Pneumokokken zurückzuführen waren. An zweiter Stelle mit 29\% lagen die durch Neisseria meningitidis bedingten Erkrankungen.

PD Dr. Johannes Liese, München, betonte, dass in Deutschland 10-15\% der Bevölkerung eine Kolonisation des Rachenraums mit Meningokokken aufweisen. Über 60\% der durch diese Erreger ausgelösten Hirnhautinfektionen werden hier durch den Serotyp B verursacht; für etwa 30\% ist Serotyp C verantwortlich, für den seit zwei Jahren eine Impfempfehlung besteht. Die Zahl der invasiven Meningokokkeninfektionen ist in den letzten Jahren bundesweit von 781 (2001) auf 436 (2007) im vergangenen Jahr deutlich zurückgegangen. Dies betrifft neben dem Serotyp C interessanterweise auch den Typ B. stoff für alle Kinder im zweiten Lebensjahr. Die Sächsische Impfkommission wie auch andere EU-Länder empfehlen Impfungen schon für Säuglinge im Alter von zwei bis zwölf Monaten. Hier erfolgen zwei Impfungen im Abstand von mindestens zwei Monaten sowie eine Auffrischungsimpfung im zweiten Lebensjahr. Alle Meningokokken-CImpfstoffe sind $a b$ dem vollendeten zweiten Lebensmonat zugelassen.

In England erfolgte bereits 1999 ein Programm gegen Menigokokken C, bei dem fast alle Säuglinge bzw. Kleinkinder bis zum Jugendlichen „durchgeimpft" wurden. Innerhalb von drei Jahren nahmen die durch Meningokokken $C$ bedingten Infektionen der unter 20-Jährigen um $92 \%$ ab. Die Mortalitätsrate sank um über $85 \%$.

\section{Vakzine gegen Subtyp B in der Pipeline}

Bislang waren die Bemühungen um eine effektive Vakzine gegen Meningokokken des Serotyps B erfolglos.

Das Unternehmen Novartis Behring hat nun in einem aufwendigen Verfah-

\section{Akuttherapie: Volumengabe wichtig}

Die Standardtherapie von Kindern mit Meningitis erfolgt mit Cefotaxim (intravenös, täglich 200 mg/kg Körpergewicht). Wichtig ist - auch für den erstversorgenden Arzt - die Einleitung einer ausreichenden Volumentherapie mit 0,9\%iger NaCl-Lösung, die zunächst als Bolusinfusion mit $20 \mathrm{ml} / \mathrm{kg}$ in fünf Minuten und danach mehrfach wiederholt mit 60-100 $\mathrm{ml} / \mathrm{kg}$ erfolgt.

Ob die Therapie mit Dexamethason wirklich effektiv ist, wird durch eine aktuelle Studie (Mongelluzzo BA, JAMA, 2008) infrage gestellt, in der sich kein Überlebensvorteil nachweisen ließ. Die zusätzliche orale Gabe von Glycerol zeigte in einer Untersuchung von Heikki Peltola und seinen Mitarbeitern signifikante präventive Effekte gegen neurologische Spätschäden (Clin Infect Dis, 2007).

$\mathrm{AFI}=$

-14. Münchner Impftag, 15. 11. 2008, Veranstalter: Dr. von Haunersches Kinderspital, Berufsverband der Kinder- und Jugendärzte e.V. und Münchner Kinderärztliche Gesellschaft, Informationen von Novartis Behring, Marburg 